\title{
Anti-ETBR/MMAE Antibody-Drug Conjugate DEDN6526A
}

National Cancer Institute

\section{Source}

National Cancer Institute. Anti-ETBR/MMAE Antibody-Drug Conjugate DEDN6526A. NCI

Thesaurus. Code C116742.

An antibody-drug conjug ate (ADC) composed of a humanized immunog lobulin (Ig) G1 monoclonal antibody ag ainst anti-endothelin B receptor (ETBR) and covalently linked to monomethyl auristatin $\mathrm{E}$ (MMAE), an auristatin derivative and a potent microtubule disrupting agent, with potential antineoplastic activity. Upon administration, the monoclonal antibody moiety of DEDN6526A binds to ETBR-expressing tumor cells and is internalized, thereby delivering MMAE intracellularly. Proteolytic cleavage releases MMAE, which then binds to tubulin and inhibits its polymerization, resulting in G2/M phase arrest and tumor cell apoptosis. ETBR, a G-protein coupled receptor that can activate RAF/MEK signaling, is overexpressed in a variety of tumor cell types and plays a key role in tumor cell proliferation, invasion, epithelial-mesenchymal transition (EMT) and ang iogenesis. 